\title{
Torsion of a non-gravid uterus: a rare cause of acute abdomen
}

\author{
Deepti Sharma*, M. G. Usha
}

Department of Obstetrics and Gynecology, Amrita Institute of Medical Sciences, Kochi, Kerala, India

Received: 27 March 2013

Accepted: 14 April 2013

\author{
*Correspondence: \\ Dr. Deepti Sharma, \\ E-mail: sdeepti108@gmail.com
}

(C) 2013 Sharma D et al. This is an open-access article distributed under the terms of the Creative Commons Attribution License, which permits unrestricted use, distribution, and reproduction in any medium, provided the original work is properly cited.

\begin{abstract}
Torsion of a non gravid uterus is a rare phenomenon. In a non-gravid uterus the torsion is usually associated with the presence of big myomas, adnexal masses, uterine anomalies and pelvic adhesions. Precise preoperative diagnosis is usually difficult and the condition is diagnosed usually during surgical exploration. Delay in diagnosis can prove fatal as the uterus and adnexa can undergo gangrenous change. Timely surgical intervention is of utmost importance in the management of such cases. We describe a case of torsion uterus in a $72 \mathrm{yr}$ old postmenopausal lady with a big fundal myoma who presented with acute abdominal pain.
\end{abstract}

Keywords: Torsion uterus, Non-gravid, Postmenopausal

\section{INTRODUCTION}

Torsion of uterus in a non gravid state is a rare clinical entity. It is defined as rotation of the uterus along its longitudinal axis by more than 45 degrees. ${ }^{1}$ Most of the cases of uterine torsion have been described in a gravid state, whereby it results in serious maternal and fetal consequences. In a non-gravid uterus the torsion is usually associated with the presence of big myomas, adnexal masses, uterine anomalies and pelvic adhesions. ${ }^{2}$ It is a potential life threatening condition and warrants urgent surgical intervention. Preoperative diagnosis is usually difficult and can be missed on a routine ultrasound.

\section{CASE REPORT}

A 75 year old, postmenopausal lady, presented to the casualty with complaints of severe abdominal pain, persistent vomiting and abdominal distension of one day duration. She had attained menopause at the age of 48 years and had no complaints in the postmenopausal period. Her obstetric history was significant for four vaginal deliveries and post partum sterilization following the last child birth.
She was dehydrated and in severe pain at the time of examination. She had mild fever, stable BP, significant tachycardia, but no pallor. Examination revealed a mass arising from the pelvis of about 18 weeks size, firm in consistency, well defined borders and tender on palpation. Cervix could not be visualized on speculum examination. On per vaginal examination cervix was pulled high up and hitched under symphysis pubis, tender on palpation, uterine body could not be made out separately.

Transabdominal ultrasound showed a solid cystic mass of 18 X 12 X $10 \mathrm{~cm}$ occupying the hypogastrium, uterine body could not be separately identified from the mass. Both ovaries also could not be localized. There was minimal free fluid in the peritoneal cavity. A provisional diagnosis of twisted ovarian tumor was made and patient was taken up for immediate laparotomy.

Abdomen was opened through midline vertical incision. Peritoneal fluid was blood stained. A large myoma with a broad attachment was seen arising from the uterine fundus (Figure 1). The uterus was rotated in longitudinal axis at the junction of corpus with the cervix, by about 270 degrees clockwise, in a manner that both the ovaries 
were facing anteriorly with the round ligament and tubal attachment cephalad and posterior to them. The specimen was detorsed in the anticlockwise direction and regained pinkish hue after few minutes (Figure 2). A total abdominal hysterectomy with bilateral salpingooophorectomy was performed. Hemostatic sutures were placed on the vault which was left open. Abdomen was closed in layers after doing a thorough lavage. Postoperative period was uneventful and patient was discharged on the fifth postoperative day. Histopathological report was confirmatory of haemorrhagic infarcts in both ovaries as well as the uterus.

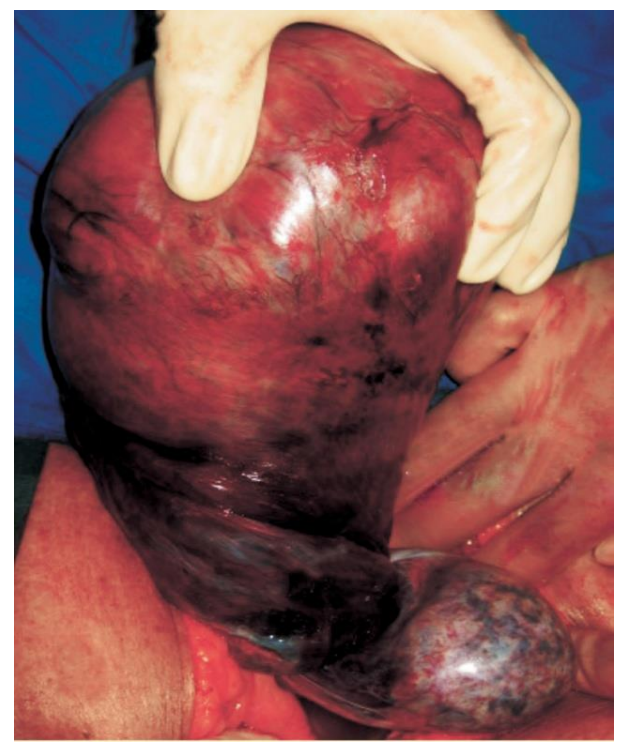

Figure 1: A $16 \mathrm{~cm}$ sessile myoma at the fundus. Both ovaries were seen anteriorly and the gonadal vessels along with tubes were greatly engorged.

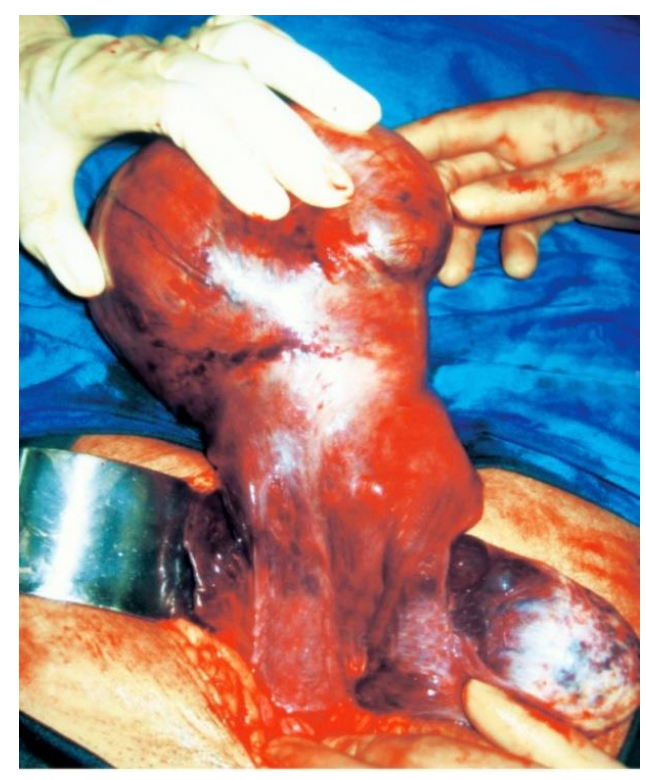

Figure 2: Uterine corpus and cervix were congested and purplish black in color. The cervix was elongated and isthmic region was gangrenous and friable.

\section{DISCUSSION}

Dextrorotation of the uterus upto 45 degrees in the gravid condition is a common occurrence. Uterine torsion is defined as rotation of more than 45 degrees on its long axis and a range of 60-900 degrees has been described. ${ }^{1}$ The rotation is clockwise in about two-third of the cases and is usually pivoted at the utero-cervical junction. ${ }^{5}$

Majority of the cases in literature are reported in pregnancy and this has been reviewed extensively by Piot et $\mathrm{al}^{3}$ and Jensen. ${ }^{4}$ According to Jensen, this condition can occur at all ages, all parity and in all the trimesters of pregnancy. The first case of torsion in the non pregnant condition was described in $1909 .{ }^{5}$ Past two decades have witnessed an increase in reports of torsion of non-gravid uterus $^{6}$ indicating more awareness about the diagnosis and increased preoperative use of advanced radiological imaging techniques.

The pathophysiology of the condition is poorly understood and unclear. Laxity and elongation of the parametrial tissues following menopause coupled with the weight of a heavy sessile myoma or an adnexal mass could be an important etiological factor. Asymmetrical enlargement of the uterus, abnormal posture and movements, irregular contraction of abdominal musculature, functional variations in size and mobility of bladder and rectum are other implicated factors. The fact that the uterine corpus has relatively weak lateral attachments as compared to the cervix, which is well anchored to the pelvic walls with cardinal and uterosacral ligaments, could be a possible explanation of torsion occurring at the level of uterine isthmus.

The presentation can be variable ranging from non specific urinary and gastrointestinal symptoms to acute abdominal pain with shock and collapse. ${ }^{7}$ Persistent abdominal pain is the most common symptom. Diagnosis can prove elusive due to vague presentation and non specific symptoms. It is extremely difficult to clinch the diagnosis on a routine ultrasound. Change in the position of a previously imaged myoma and abnormal position of ovarian vessels across the uterus using colour doppler can aid the diagnosis on ultrasound. CT finding of whorled appearance of the cervix has been described by Luk et $\mathrm{al}^{8}$ indicative of twisting of cervix. Nicholson et $\mathrm{al}^{9}$ have suggested X-shaped configuration of upper vagina on MRI as a sign to diagnose torsion, which is normally visualized as $\mathrm{H}$ shaped structure.

The condition demands immediate surgical exploration as the uterus and adnexa can undergo gangrene if the diagnosis is delayed. The surgical options are influenced by the age of the patient, desire to preserve fertility, associated pathology and viability of the uterus and adnexae. Total hysterectomy with bilateral adnexectomy is usually performed in elderly women and especially if uterus has undergone necrosis due to prolonged torsion.,7 Clear orientation of anatomy is essential in order to avoid 
an inadvertent injury to the ureter and gonadal vessels. If conservative surgery is aimed in younger age group, it usually includes myomectomy or ovarian cystectomy, along with plication of round or uterosacral ligaments.

\section{CONCLUSION}

Although uterine torsion is a rare condition, it should be considered as an important differential diagnosis in women with a big myoma or adnexal mass presenting with acute abdominal pain. The diagnosis can come as a surprise during exploratory laparotomy as the preoperative diagnosis is usually thought to be a twisted ovarian cyst or a twisted pedunculated myoma. Delay in diagnosis can prove fatal and prompt surgical intervention is crucial in the management of such cases.

\section{Funding: None}

Competing interests: None declared

Ethical approval: Not required

\section{REFERENCES}

1. Collinet P, Narducci F, Stien L. Torsion of a nongravid uterus: an unexpected complication of an ovarian cyst. Eur J Obstet Gynecol Reprod Biol 2001;98:256-7.
2. Jeong YY, Kang HK, Park JG, et al. CT features of uterine torsion. Eur Radiol 2003;13:249-50.

3. Piot D, Gluck M, Oxorn H. Torsion of gravid uterus. Can Med Assoc J 1973;109:1010-1.

4. Jensen JG. Uterine torsion in pregnancy. Acta Obstet Gynecol Scand 1992;71:260-5.

5. Hawes $\mathrm{CH}$. Acute axial torsion of the uterus. Ann Surg 1935;102:37-40.

6. Attapattu JA, Prussia PR, Menon S. Torsion of a non-pregnant fibromyomatous uterus. Int J Gynaecol Obstet 1994;45:163-4.

7. Dua A, Fishwick K, Deverashetty B. Uterine torsion in pregnancy: a review. The Internet Journal of Gynecology and Obstetrics 2006;6(1).

8. Luk SY, Leung JL, Cheung ML, et al. Torsion of a nongravid myomatous uterus: radiological features and literature review. Honk Kong Med J 2010;16:304-6.

9. Nicholson WK, Coulson CC, McCoy MC, Semelka RC. Pelvic magnetic resonance imaging in the evaluation of uterine torsion. Obstet Gynecol 1995;85:888-90.

DOI: $10.5455 / 2320-1770 . i j r \operatorname{cog} 20130628$

Cite this article as: Sharma D, Usha MG. Torsion of a non-gravid uterus: a rare cause of acute abdomen. Int J Reprod Contracept Obstet Gynecol 2013;2:234-6. 